\title{
Heterogeneity and Clinical Significance of Glomerular-binding Antibodies in Systemic Lupus Erythematosus
}

\author{
James B. Lefkowith, ${ }^{\star}$ Mary Kiehl, ${ }^{\star}$ Joel Rubenstein,, ${ }^{\star}$ Richard Di Valerio, ${ }^{\star}$ Keith Bernstein, ${ }^{\star}$ Leslie Kahl, ${ }^{\star}$ Robert L. Rubin, ${ }^{\S}$ \\ and Mark Gourley $¥$ \\ *Departments of Medicine and Molecular Biology and Pharmacology, Washington University School of Medicine, St. Louis, Missouri \\ 63110; ${ }^{\ddagger}$ National Institutes of Arthritis and Musculoskeletal and Skin Diseases, National Institutes of Health, Bethesda, Maryland 20892; \\ and ${ }^{\S}$ Department of Molecular and Experimental Medicine, The Scripps Research Institute, La Jolla, California 92037
}

\begin{abstract}
We used an ELISA employing extracts of human glomerular basement membrane (GBM) to detect, characterize, and evaluate the clinical significance of glomerular-binding IgG in patients with SLE nephritis. Most patients with SLE nephritis exhibited GBM-binding IgG, although many patients with active nonrenal SLE or symptomatic, drug-induced lupus had similar reactivity, albeit at lower levels. IgG binding to GBM in SLE nephritis patients was decreased by DNase pretreatment of GBM, restored after DNase with nuclear antigens (most notably with nucleosomes), inhibited by exogenous nuclear antigens (particularly nucleosomes), but unaffected by exposure of serum to DNase/high ionic strength. The characteristics of IgG binding to GBM largely paralleled the patients' underlying autoimmune response, which was dominated either by antibodies to DNA/nucleosomes or to nucleosomes alone. Binding of lupus sera to nonrenal extracellular matrix (even with nucleosomes) was not equivalent to GBM. Collagenase pretreatment of GBM variably decreased IgG binding, depending on the level and type of binding. SLE nephritis patients with high levels of GBM-binding IgG exhibited more severe disease clinically, but the same renal histopathology, as patients with lower levels. The level of GBM-binding IgG at presentation did not predict the therapeutic response, but decreased in responders to therapy. In sum, glomerular-binding IgG in lupus nephritis binds to epitopes on chromatin, which adheres to GBM in part via collagen. These autoantibodies appear necessary, but not sufficient, for the development of nephritis, and correlate with clinical rather than histopathologic parameters of disease activity. (J. Clin. Invest. 1996. 98: 1373-1380.) Key words: lupus nephritis - autoantibodies • glomerular basement membrane $\bullet$ systemic lupus erythematosus $\bullet$ nucleosomes
\end{abstract}

\section{Introduction}

The prevailing wisdom regarding SLE nephritis is that this disorder is mediated by anti-DNA antibodies (reviewed in refer-

Address correspondence to James B. Lefkowith, Box 8045, Division of Rheumatology, Washington University School of Medicine, St. Louis, MO 63110. Phone: 314-362-8601; FAX: 314-362-0419; E-mail: jlefkowith@imgate.wustl.edu

Received for publication 15 April 1996 and accepted in revised form 10 July 1996.

The Journal of Clinical Investigation

Volume 98, Number 6, September 1996, 1373-1380 ence 1). The presence of anti-DNA antibodies correlates with nephritis in both mice and human patients, anti-DNA antibodies are concentrated within human and murine glomeruli in vivo, administration of DNA to autoimmune mice accelerates the progression of nephritis, and anti-DNA mAbs produce nephritis by adoptive transfer (2-9). Substantial data have accumulated which suggest, however, that anti-DNA antibodies are neither necessary nor sufficient for nephritis to occur (reviewed in reference 1). Immunogenetic studies of murine lupus have demonstrated that not all mice with anti-DNA antibodies develop nephritis and that nephritis can occur in their absence $(10,11)$. Only certain anti-DNA mAbs are pathogenic when administered to nonautoimmune mice $(7,9)$. Moreover, patients with lupus nephritis do not invariably have anti-DNA antibodies $(3,12,13)$.

Apropos to these issues, recent studies have reinforced the concept that SLE is an antichromatin disease; e.g., that the autoimmune response is against chromatin with the development of antibodies directed to various epitopes on chromatin including but not limited to DNA (14-19). The possibility that antinucleosomal antibodies may generally be involved in nephritis has also been suggested by recent experimental and clinical studies $(15,18,20,21)$. In one recent clinical study of SLE, antibodies to epitopes on chromatin were found to be more highly associated with nephritis than antibodies to DNA (15).

The concept that lupus nephritis involves the participation of a family of antinucleosomal antibodies has also been supported by recent work by our group on identifying glomerulotropic antibodies in lupus nephritis. In our studies, we used glomerular and glomerular basement membrane $(\mathrm{GBM})^{1}$ binding ELISAs to define glomerular-binding antibodies in murine lupus serum and to produce glomerular-binding mAbs. The antibodies detected by these assays in MRL lpr serum react specifically with glomeruli in vitro, concentrate in glomeruli in vivo, and correlate with the presence of nephritis $(9,22$, 23). Analysis of MRL lpr serum and mAbs derived from these mice has demonstrated that such antibodies comprise a family of IgGs that react with various epitopes on chromatin adherent to GBM type IV collagen $(24,25)$. These data support the hypothesis that the immunopathogenesis of lupus nephritis involves the contribution of multiple autoantibodies that bind to the glomerulus by virtue of chromatin adherence to GBM collagen.

In other recent work, we have found that glomerular-binding antibodies are associated with nephritis in SLE patients (26). The intent of the current work was to characterize these

1. Abbreviations used in this paper: dsDNA, double-stranded DNA; ECM, extracellular matrix; GBM, glomerular basement membrane; $\mathrm{H}$, histone. 
human glomerular-binding antibodies, and thereby test the above hypothesis for human SLE. Towards this end, we developed a human GBM ELISA similar to that which we used in our murine studies $(24,25)$. We used this assay to characterize glomerulotropic antibodies in lupus patients, and to assess both the diagnostic and prognostic import of such antibodies. The results we obtained suggest that we should reorient our conceptualization of lupus nephritis to a disease mediated by antichromatin autoantibodies rather than simply anti-DNA antibodies. Moreover, the data suggest that the development of nephritis is not simply a function of the presence of these autoantibodies.

\section{Methods}

Patient characteristics and treatment. Sera were obtained from 63 patients with established lupus nephritis who were enrolled in a randomized prospective study at the National Institutes of Health. Entry required $(a)$ a diagnosis of SLE based on the American College of Rheumatology classification criteria (27), and (b) glomerulonephritis. Glomerulonephritis was defined as an active urine sediment $(\geq 10$ red blood cells/hpf and/or cellular casts without evidence of infection on two or more urinalyses) or $>3 \mathrm{~g}$ proteinuria/24 h plus histological evidence of proliferative glomerulonephritis on a renal biopsy obtained within 3 mo of study entry. Two patients were not biopsied because of uncontrolled hypertension or anticoagulation therapy, but both patients exhibited an active sediment and $>3 \mathrm{~g}$ proteinuria/24 $\mathrm{h}$.

Sera from 15 patients with clinically active nonrenal lupus and from 33 patients with clinically inactive lupus were obtained from patients in the Rheumatology Clinic at Washington University. These patients also fulfilled the American College of Rheumatology criteria for SLE (27). The former patients had clinically active disease requiring treatment at the time of sample procurement, but did not fulfill the American College of Rheumatology criterion for nephritis. The latter patients had no evidence of active lupus at the time of sample procurement and for $1 \mathrm{yr}$ thereafter. Patients with symptomatic, drug-induced lupus and with drug-induced autoantibodies without symptoms have been described in detail $(28,29)$. The patients with symptomatic, drug-induced lupus included eight patients with lupus induced by procainamide, six by hydralazine, seven by quinidine, and one each with lupus induced by acebutalol, penicillamine, methyldopa, and timolol. All asymptomatic patients were treated with procainamide for an average of $2 \mathrm{yr}$ and had elevated antibody activity to denatured DNA and/or denatured histones. Normal sera were obtained from eight healthy volunteers.

Lupus nephritis patients were randomized to one of three treatment regimens, the details of which have been preliminarily reported elsewhere (30). A response to therapy was defined as concurrent fulfillment of all three of the following criteria: clearing of the urine sediment ( $<10$ red blood cells/hpf, no cellular casts), proteinuria $<1.0 \mathrm{~g} / \mathrm{d}$, and a serum creatinine more than twofold greater than the entry value. Nonresponder status was defined as a failure to meet all of these criteria.

Detection of glomerular-binding antibodies. We used a human GBM ELISA to detect glomerular-binding antibodies in human sera. We have previously established that the murine GBM and glomerular ELISAs detect the same set of antibodies (24). Human GBM was isolated from glomeruli obtained from normal human kidneys (provided by MidAmerica Transplant Association, St. Louis, MO) and used to establish an ELISA, as we have published for murine GBM (24). Assay values were unaffected by the use of different lots of GBM (data not shown). As previously noted with murine GBM, nuclear antigens are present in the substrate when isolated (24). The acquisition of nuclear antigens probably occurs with substrate preparation, since immunohistochemical studies suggest that nuclear antigens are present in glomeruli from lupus nephritis patients, but not normal controls (31-33). As positive controls for GBM binding, we used both an anti-GBM antiserum (Binding Site Inc., San Diego, CA) and a reference serum from our lupus nephritis serum bank with binding activity to nucleosomes, DNA, and histones. All sera were assayed at a 1:100 dilution, except for the anti-GBM antiserum, which was diluted to 1:50. The anti-GBM serum yielded a signal of $0.297 \pm 0.008$ OD and the reference serum yielded a signal of $0.745 \pm 0.014$ OD.

We used an analogous ELISA format to determine binding to human placental extracellular matrix (ECM) and human type IV collagen (Collaborative Biomedical Products, Bedford, MA). Human placental ECM is similar to GBM in composition since it contains laminin, type IV collagen, and heparan sulfate proteoglycan. Binding to placental ECM was also determined in the presence of an additional source of nuclear antigen by sequentially coating ELISA plates with $40 \mu \mathrm{g} / \mathrm{ml}$ placental ECM and $10 \mu \mathrm{g} / \mathrm{ml}$ nucleosomes (Worthington Biochemical Corp., Freehold, NJ).

To examine the role of chromatin or collagen in GBM binding, the GBM was enzymatically treated before use in the assay. For these experiments GBM-coated plates were exposed either to $10 \mu \mathrm{g} / \mathrm{ml} \mathrm{hu}$ man recombinant DNase type I (Genentech, San Francisco, CA) in PBS with $\mathrm{MgCl}_{2} 5 \mathrm{mM}$ or $300 \mathrm{U} / \mathrm{ml}$ Achromobacter collagenase (Sigma Immunochemicals, St. Louis, MO), using previously described protocols (24). Enzymatically treated wells were washed before exposure to the primary antibody. Because histones may bind to GBM components (heparan sulfate proteoglycan, type IV collagen) with high affinity $(34,35)$, in certain experiments, we exposed GBM to buffer of high ionic strength after DNase to remove residual histones. For these experiments, GBM was treated with $8 \mathrm{M} \mathrm{NH}_{4} \mathrm{SCN}$ for $15 \mathrm{~min}$ after DNase. We have observed that this concentration is necessary to dissociate histones from type IV collagen in vitro, but does not disrupt the substrate (data not shown).

To determine the nature of IgG binding to GBM, reconstitution and inhibition studies using exogenous nuclear antigens were performed. In the reconstitution studies, GBM was treated with DNase and then incubated with one of the following nuclear antigens at 10 $\mu \mathrm{g} / \mathrm{ml}$ before assay: histones (purified mixture of $\mathrm{H} 1, \mathrm{H} 2 \mathrm{~A}, \mathrm{H} 2 \mathrm{~B}, \mathrm{H} 3$, and H4; Calbiochem-Novabiochem Corp., La Jolla, CA), doublestranded (ds)DNA ( $\lambda$ phage DNA; Sigma), or nucleosomes (Worthington), as previously described (24). In the inhibition studies, serum was preincubated with histones, dsDNA, or nucleosomes (at a final concentration of $100 \mu \mathrm{g} / \mathrm{ml}$ ) before assay, as described (24).

To determine a potential contribution of immune complexes to GBM or DNA-binding IgG in lupus sera, sera were exposed to conditions of high ionic strength and DNase simultaneously before assay using a variation of a published method (20). Serum was diluted 1:5 (vol:vol) in $3 \mathrm{M} \mathrm{NaCl}$ containing $\mathrm{MgCl}_{2} 5 \mathrm{mM}$ and $100 \mu \mathrm{g} / \mathrm{ml}$ DNase, and incubated $16 \mathrm{~h}$ at $37^{\circ} \mathrm{C}$. Before assay, serum was further diluted in $1 \mathrm{mM}$ EDTA containing $10 \mathrm{mg} / \mathrm{ml} \mathrm{BSA}$ to inactivate the DNase, achieve isotonicity, and produce a final dilution of 1:100 for assay. The binding of DNase/high ionic strength-treated serum was compared to that of untreated serum. DNase was active in the high ionic strength buffer (data not shown).

Anti-dsDNA and antinuclear antigen ELISAs. The anti-dsDNA assay was performed using a previously published ELISA protocol that uses $\lambda$ phage dsDNA coated on poly-L-lysine (Sigma; 23). Binding to intact chromatin, oligonucleosomes (2-8 nucleosomes in length, stripped of histone H1), histone H2A-H2B-DNA complexes, histone (H3-H4) ${ }_{2}$-DNA complexes, histone H2A-H2B complexes without DNA, histone (H3-H4) $)_{2}$ complexes without DNA, or individual histones ( $\mathrm{H} 1, \mathrm{H} 2 \mathrm{~A}, \mathrm{H} 2 \mathrm{~B}, \mathrm{H} 3$, and $\mathrm{H} 4)$ were also performed by ELISA as published (25). Individual histones used as assay substrates were purchased from Calbiochem-Novabiochem, while other nuclear antigens were prepared as described (36).

Statistical analysis. GBM and nuclear antigen ELISAs were run in duplicate and are presented as average values. GBM reconstitution and inhibition studies were performed in triplicate. Data for these experiments were normalized to the GBM binding of unmanipulated serum $(=100 \%)$ to facilitate comparisons between patients. Regres- 
sion analysis of nuclear antigen reactivity and GBM binding was performed using the least squares method. Comparisons of clinical data between the subgroups of patients with lupus nephritis were performed by Mann-Whitney $U$ test, and median values are shown. Comparison of multiple groups used one-way ANOVA combined with Student's $t$ test, since analysis was restricted to a limited number of comparisons. Comparison of two groups used Student's $t$ test. Means \pm SEM are shown for the groups.

\section{Results}

Quantification of GBM-binding antibodies in patients with $S L E$. As shown in Fig. 1, the average level of IgG binding to GBM in lupus nephritis patients was substantially and significantly greater than that observed in normal individuals. Virtually all lupus nephritis patients (81\%) exhibited GBM-binding $\mathrm{IgG}$ greater than the normal range (i.e., $>0.05 \mathrm{OD})$. The average IgG binding to GBM in lupus nephritis patients was also significantly greater than that in patients with inactive lupus, although a number of patients with inactive lupus also exhibited levels greater than normal patients (Fig. 1). As indicated above, none of the patients with inactive lupus (even with elevated levels of GBM-binding $\mathrm{IgG}$ ) developed nephritis during the next year. Additionally, levels of GBM-binding IgG did not correlate with a previous history of nephritis in these pa-

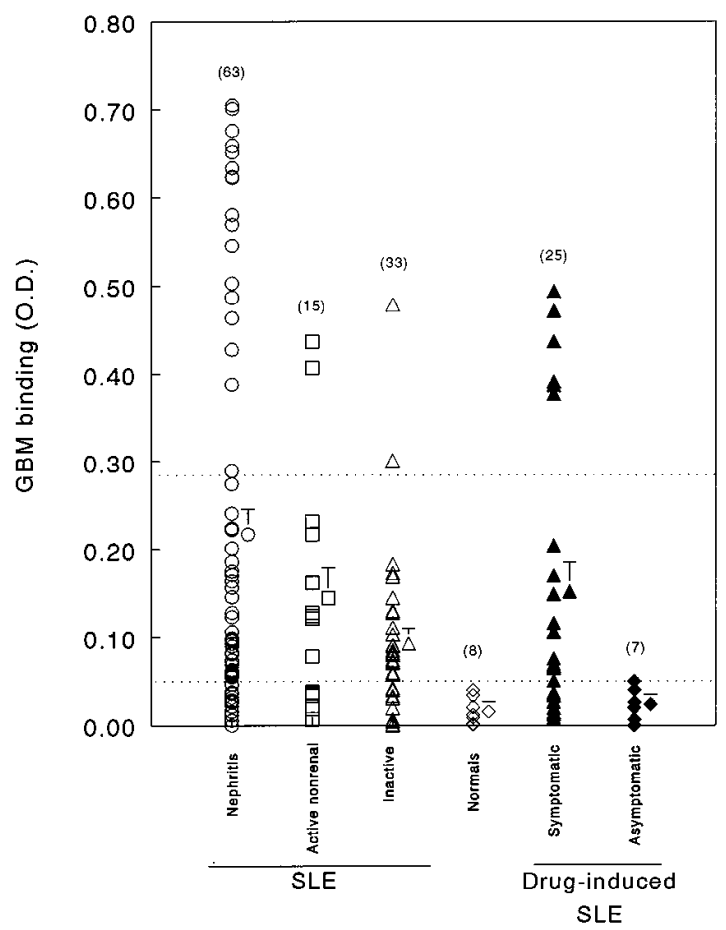

Figure 1. GBM-binding $\operatorname{IgG}$ in lupus nephritis patients. $\operatorname{IgG}$ binding to human GBM was determined by ELISA in the sera of the six groups of patients shown. The means \pm SEM for the groups are displayed to the right of the individual values. The dotted lines represent the upper limits of the ranges (mean $+2 \times$ SDs) defined by the inactive lupus patients and normal controls (upper and lower lines, respectively). Lupus nephritis patients were significantly greater than inactive lupus patients, normal controls, and patients with asymptomatic, drug-induced lupus $(P<0.0001$ for each comparison). A trend towards a difference between lupus nephritis patients and patients with active, nonrenal lupus $(P=0.109)$ and symptomatic, drug-induced lupus $(P=0.142)$ was also noted. tients (data not shown). The average level of GBM-binding IgG in patients with lupus nephritis was not significantly greater than that in patients with active lupus but without clinical evidence of renal disease (Fig. 1).

We additionally quantified GBM-binding $\operatorname{IgG}$ in the sera of patients with drug-induced lupus. As shown in Fig. 1, the average level of GBM-binding $\mathrm{IgG}$ in patients with symptomatic, drug-induced lupus was similar to that in patients with active lupus without renal disease and was not significantly different from that in patients with SLE nephritis. In contrast, patients with drug-induced SLE without symptoms (i.e., asymptomatic but serologically abnormal) exhibited an average level of GBM-binding IgG similar to normal controls (Fig. 1).

Qualitative characterization of GBM-binding IgG in lupus nephritis patients. We used the group of inactive lupus patients to separate patients with active lupus nephritis into two groups: those with high GBM-binding IgG $(>$ mean $+2 \times$ SD of the inactive population or $>0.285$ OD) and those with lower levels $(<0.285$ OD). $27 \%$ of the nephritis patients fell into the former category. Qualitative characterization of the GBM-binding IgG in representative patients with high levels demonstrated substantial similarities among patients. GBM binding was uniformly diminished by DNase pretreatment of the GBM (average decrease of $67 \pm 6 \%$, Fig. $2 A$ ). In contrast, DNase did not disrupt the binding of the commercial antiGBM antibody (data not shown). Binding of these sera was also efficiently reconstituted by incubating the DNase-treated GBM with either dsDNA alone or nucleosomes before assay (Fig. 2 A). In several patients (Nos. 25, 100, and 106), however, histones alone were able to reconstitute binding, although less efficiently than either DNA or nucleosomes (Fig. $2 A$ ). Inhibition studies using exogenous nuclear antigens largely corroborated the reconstitution studies (Fig. $2 \mathrm{~B}$ ). Nucleosomes and dsDNA consistently inhibited binding, whereas the effect of histones was more modest and variable.

Profiling the nuclear antigen-binding characteristics of the sera from these patients demonstrated an overall parallelism between the characteristics of IgG binding to GBM and the underlying autoimmune response to nuclear antigens (Fig. 2 $C$ ). The autoimmune response of this group of patients was characterized by substantial binding to dsDNA, chromatin, and subnucleosomes. Many of these patients, however, exhibited considerable binding to individual histones or histone-histone complexes. Although no common histone-binding pattern was observed among patients, the ability of histones to reconstitute GBM binding after DNase treatment in this group of patients was correlated with reactivity to the $\mathrm{H} 2 \mathrm{~A}-\mathrm{H} 2 \mathrm{~B}$ dimer ( $r=0.85, P=0.017$ for the correlation between histone reconstitution of GBM binding and $\mathrm{H} 2 \mathrm{~A}-\mathrm{H} 2 \mathrm{~B}$ binding; $P>$ 0.05 for other correlations).

The qualitative characteristics of GBM-binding $\mathrm{IgG}$ from representative patients with lower levels of such IgG were distinct from this first group. In these patients, GBM binding was comparably disrupted by DNase pretreatment of the GBM (average decrease in binding of $64 \pm 9 \%$, Fig. $3 A$ ). Binding was reconstituted most consistently and effectively with nucleosomes, however, and less efficiently (and more variably) with histones or dsDNA alone (Fig. $3 A$ ). Inhibition studies in these patients exhibited two different patterns (Fig. $3 B$ ). One subset of these patients (Nos. 16, 71, 96, and 117) exhibited inhibition with nucleosomes and DNA, and variable inhibition with histones. In contrast, a second subset of patients (Nos. 77, 86, and 
102) exhibited little inhibition (and occasionally enhanced binding) in these experiments. These latter patients were those who exhibited the most exuberant reconstitution of GBM binding with histones.

Similar to the patients with high levels of GBM-binding $\mathrm{IgG}$, an overall parallelism between $\mathrm{IgG}$ binding to GBM and the underlying autoimmune response to nuclear antigens was observed in patients with lower levels (Fig. $3 \mathrm{~B}$ ). The autoim-

A

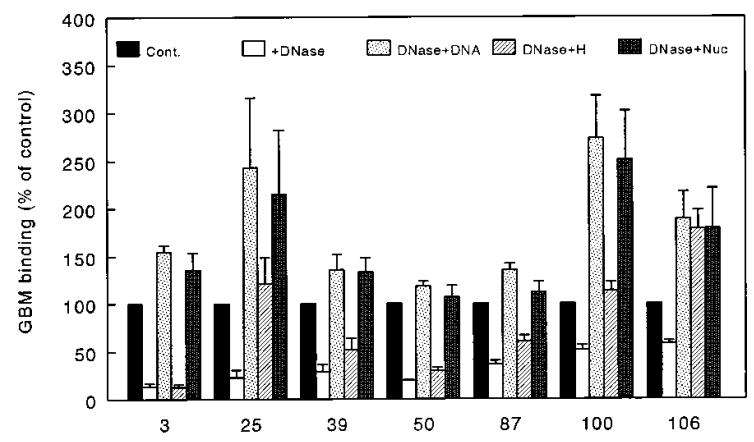

B

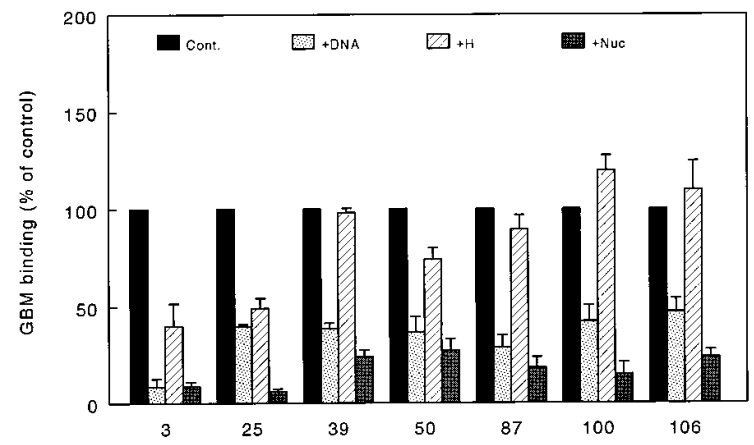

C

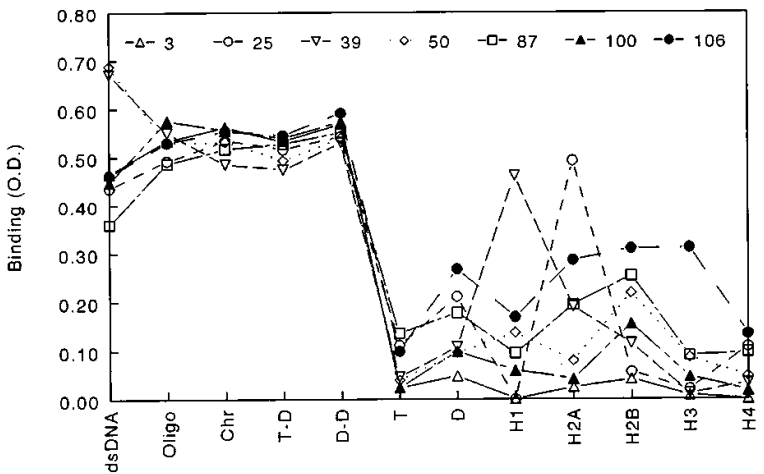

Figure 2. Characterization of GBM binding and nuclear antigen reactivity in sera from patients with GBM-binding IgG $>0.285$ OD. The characteristics of IgG binding to GBM in representative patients with GBM-binding IgG $>0.285$ OD was determined using both reconstitution and inhibition strategies. GBM binding is normalized to $100 \%$, and individual patients are referred to by number on the $x$ axis. The binding of these same sera to a panel of defined nuclear antigens was concurrently determined. (A) Effect of DNase pretreatment of GBM on binding, and the ability of nuclear antigens to reconstitute binding after DNase. (B) Inhibition of GBM binding by exogenous nuclear antigens. $(C)$ Nuclear antigen binding. Nuc, nucleosomes; Oligo, oligonucleosomes; Chr, chromatin; T-D, H3-H4 tetramers with DNA; D-D, H2A-H2B dimers with DNA; T, H3-H4 tetramers; D, H2A-H2B dimers. mune response in these latter sera was dominated by uniform reactivity with chromatin and subnucleosomes with modestto-negligible reactivity to dsDNA. The binding of these sera to individual histones or histone-histone complexes was also modest with the exception of binding to $\mathrm{H} 1$ in several of the sera. No correlation between the ability of histones to reconstitute GBM binding and binding to individual histones or histone-histone complexes was observed ( $P>0.05$ for all correlations).

Because DNase treatment of GBM did not completely prevent the binding of IgG from lupus nephritis sera, we conjec-

A

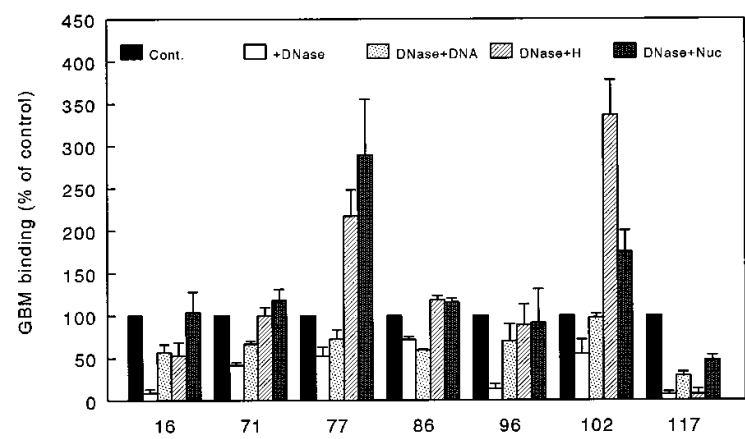

B

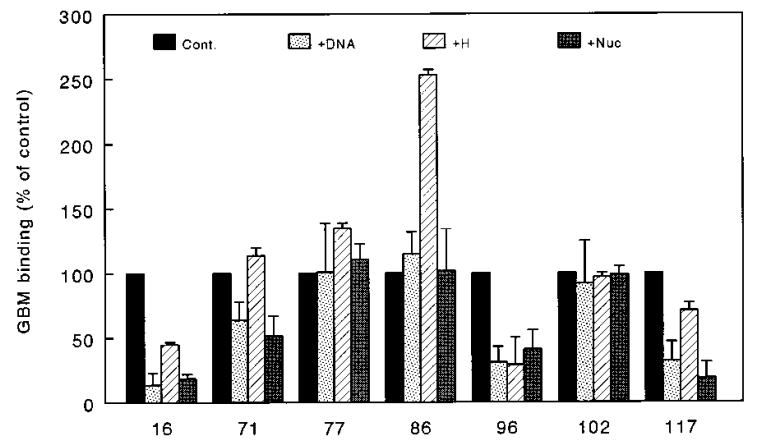

C

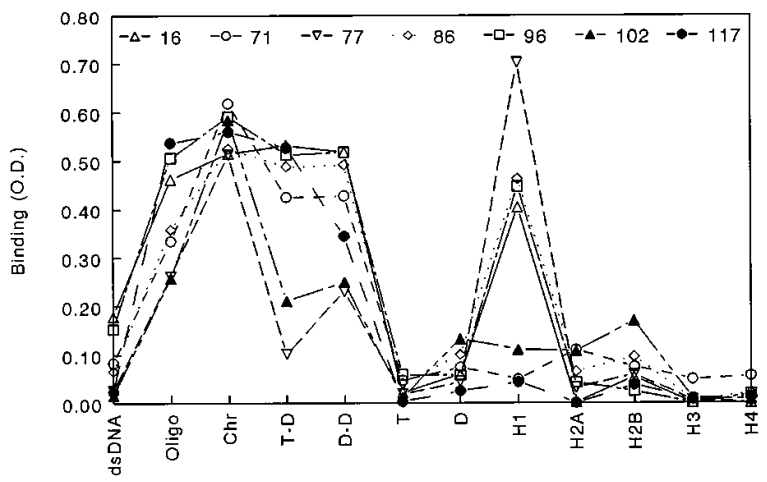

Figure 3. Characterization of GBM binding and nuclear antigen reactivity in sera from patients with GBM-binding $\operatorname{IgG}<0.285 \mathrm{OD}$. The characteristics of $\mathrm{IgG}$ binding to GBM in representative patients with GBM-binding IgG $<0.285$ OD was determined using both reconstitution and inhibition strategies. GBM binding is normalized to $100 \%$, and individual patients are referred to by number on the $x$ axis. The binding of these same sera to a panel of defined nuclear antigens was concurrently determined. $(A)$ Effect of DNase pretreatment of GBM on binding and the ability of nuclear antigens to reconstitute binding after DNase. (B) Inhibition of GBM binding by exogenous nuclear antigens. $(C)$ Nuclear antigen binding. 
tured that this residual binding might be caused by immune complexes binding to GBM (e.g., anti-DNA antibodies complexed to DNA or nucleosomes; 20, 25, 37). To test for this possibility, we exposed serum from patients with high levels of GBMbinding $\mathrm{IgG}$ to high ionic strength/DNase, and then examined for an effect on binding to GBM, DNA, or poly-L-lysine (precoat for the anti-DNA assay). This manipulation, however, did not significantly alter binding to these substrates (data not shown). We subsequently postulated that the residual binding after DNase might be caused by retained histones that can bind to GBM with high affinity $(34,35)$. Consequently, we examined the effect of DNase followed by high ionic strength on GBM binding. Using sera with high GBM-binding IgG, we observed that the combination of these two treatments abrogated GBM binding (decrease of $96 \pm 2 \%, n=7$ ).

To examine the specificity of GBM binding, we next determined whether nonrenal ECM could substitute for GBM in the binding assay. As shown in Fig. 4, lupus nephritis patients, patients with active nonrenal lupus, and patients with inactive lupus were differentiated by their respective levels of GBMbinding IgG, but not by the level of IgG binding to placental ECM (which is similar in composition to the GBM, see Methods). The inability of placental ECM to substitute for GBM could not be rectified by adding nucleosomes to the placental ECM substrate (Fig. 4).

As we have noted with murine lupus, type IV collagen within the GBM is necessary for autoantibody binding because of the adherence of nuclear antigens to this protein $(24,25)$. Autoantibody binding to type IV collagen per se is negligible $(24,25)$. We tested these statements in human lupus by examining the effect of collagenase on GBM binding of lupus nephritis sera. As shown in Fig. 5, GBM binding of lupus nephritis sera was generally decreased by preincubation of the GBM with collagenase, though substantial variability was noted among patients (ranging from a decrease of 0 to $100 \%$ ). The

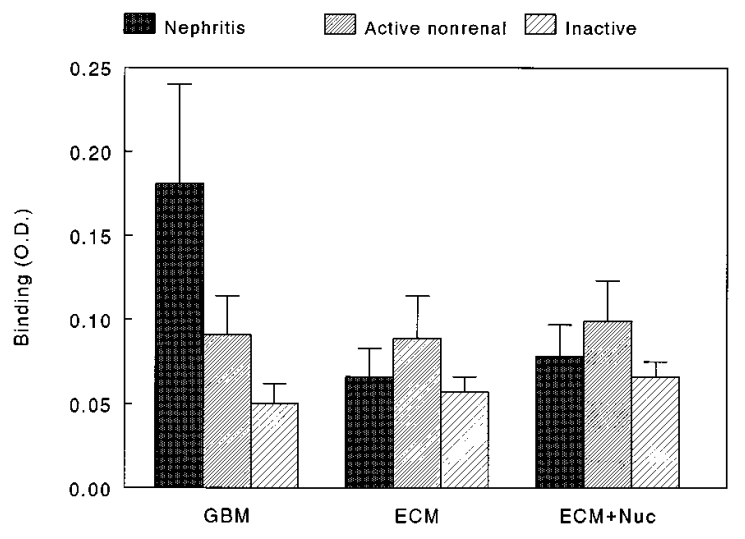

Figure 4. IgG binding of lupus sera to GBM vs. nonrenal ECM. IgG binding to GBM, placental ECM (without or with added nucleosomes $[\mathrm{Nuc}]$ ) was determined by ELISA in sera from lupus patients with nephritis, active nonrenal disease, and inactive disease $(n=15$ for each group). For the GBM assay, lupus nephritis patients were significantly greater than lupus patients with inactive disease $(P=$ $0.004)$, with a trend towards a difference between lupus nephritis patients and patients with active nonrenal lupus $(P=0.163)$ similar to the larger analysis shown in Fig. 1. For the placental ECM assay (run without or with added nucleosomes), no significant differences were noted.

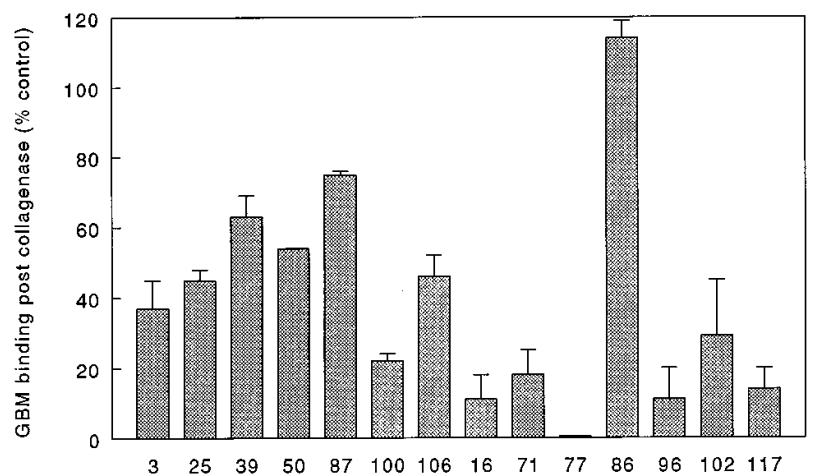

Figure 5. Effect of collagenase pretreatment of GBM on IgG binding from lupus nephritis sera. The binding of IgG from lupus nephritis sera to untreated and collagenase-pretreated GBM extracts was determined and normalized to the binding to untreated GBM (=100\%). Sera were from the patients characterized in Figs. 2 and 3 (patient number on the $x$ axis).

average decrease in a set of 25 lupus nephritis sera (14 with GBM binding $>0.285$ and 11 with GBM binding $<0.285$ OD) was $65 \pm 5 \%$. The effect of collagenase on GBM binding tended to be less substantial in sera with GBM binding $>0.285$ OD vs. sera with GBM binding $<0.285$ OD: $57 \pm 5 \%$ vs. $76 \pm 9 \%$ inhibition, respectively $(P=0.057)$. Such sera could also be distinguished, based on the characteristics of IgG binding to GBM (see above) and clinical associations (see below). Lupus nephritis sera did not bind to human type IV collagen per se (data not shown).

Association of GBM-binding $\operatorname{IgG}$ in lupus nephritis patients with clinical parameters and renal histopathology. We next sought to determine whether the level of GBM-binding IgG (which, as noted above, was correlated with the overall characteristics of IgG binding to GBM) could be related to the clinical status, histopathology, or prognosis of the patient. Patients with high levels of GBM-binding IgG overall had evidence of more severe disease by clinical criteria than patients with lower levels of GBM-binding IgG (Table I). The former group of patients

Table I. Clinical Parameters in Lupus Nephritis Patients Subdivided by GBM-binding IgG Level

\begin{tabular}{lccc}
\hline \multicolumn{1}{c}{ Parameter } & $\begin{array}{c}\text { GBM }>0.285 \\
(n=17)\end{array}$ & $\begin{array}{c}\text { GBM }<0.285 \\
(n=46)\end{array}$ & $P$ \\
\hline C3 & 60.1 & 72.9 & 0.0348 \\
C4 & 9.6 & 15.7 & 0.0802 \\
CH50 & 19.9 & 47.3 & 0.0004 \\
Creatinine clearance & 61.4 & 76.2 & 0.0750 \\
Serum creatinine & 1.42 & 1.24 & 0.3000 \\
Proteinuria & 3.72 & 4.33 & 0.5861 \\
ESR & 85.6 & 60.7 & 0.0114 \\
Hematocrit & 30.3 & 34.4 & 0.0155 \\
IgG & 1,541 & 1,034 & 0.0202 \\
Renal histology & WHO III-3 & WHO III-10 & \\
& WHO IV-13 & WHO IV-35 & \\
& & & \\
\end{tabular}

Lupus nephritis patients were subdivided into two groups, depending on whether or not the GBM-binding IgG was greater or less than the range defined by the inactive lupus patients $(>$ or $<0.285$ OD). 


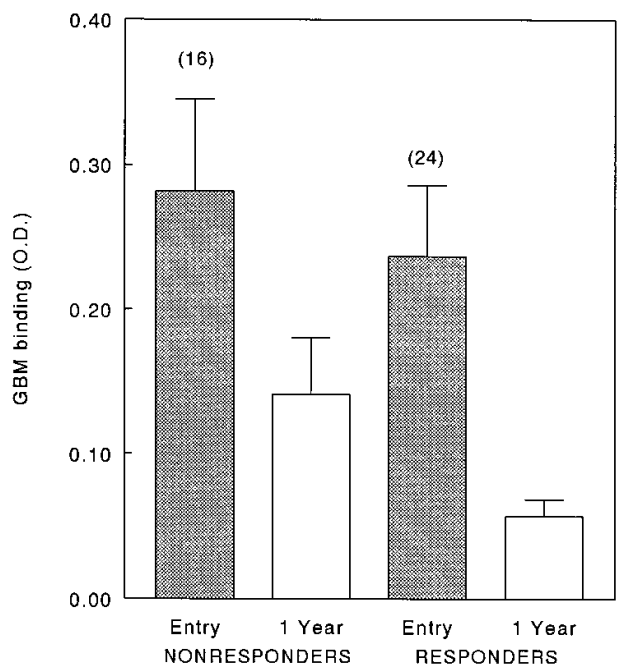

Figure 6. Effect of therapy on GBM-binding IgG in lupus nephritis patients. Patients with lupus nephritis were subgrouped depending on whether or not they exhibited a successful response to therapy (i.e., responders and nonresponders). GBM-binding $\operatorname{IgG}$ values for these two groups before and after therapy are shown. The GBM-binding $\mathrm{IgG}$ after therapy in the responders was significantly decreased $(P<$ $0.05)$.

exhibited evidence of more substantial complement activation in vivo with lower serum levels of $\mathrm{C} 3$ and $\mathrm{C} 4$, and a more severely depressed $\mathrm{CH} 50$ than the latter group. These patients also exhibited higher erythrocyte sedimentation rates, higher levels of $\mathrm{IgG}$, and lower hematocrits on average. Renal function was diminished in both groups of patients, with a trend toward more severe renal dysfunction in the former group. Despite these differences, the overall histopathologic severity was similar in the two groups (Table I). Proportionate numbers of patients with diffuse proliferative nephritis (WHO class IV) and focal/segmental proliferative glomerulonephritis (WHO class III) were seen in the two groups.

We additionally segregated the cohort of patients into subgroups, depending on their subsequent response to therapy after 1 yr to analyze the relationship between the amount (and consequently type) of GBM-binding IgG and prognosis (Fig. $6)$. There was no significant difference in the average level of GBM-binding IgG before therapy in those patients responding to therapy vs. those patients who were refractory to therapy. Patients who were successfully treated exhibited a significant decrease in GBM-binding IgG, however, while those patients who were not successfully treated exhibited a more modest (and not statistically significant) decrease in GBM-binding IgG.

\section{Discussion}

This study demonstrates the potential heterogeneity in glomerulotropic antibodies in SLE patients. As we observed, one subgroup of patients with lupus nephritis exhibited GBMbinding IgG that bound largely in a DNA- and nucleosomedependent fashion. A subset of these patients also had GBM binding that was histone dependent. A second subgroup of patients exhibited GBM-binding IgG that bound largely in a nucleosome-dependent fashion. These data support our contention derived from an analysis of murine lupus that lupus nephritis is immunologically complex, specifically that multiple antibodies (including but not limited to anti-DNA antibodies) may contribute to nephritis $(24,25)$.

Recent observations suggest that SLE is characterized by a breakdown of tolerance to chromatin $(14,15,17)$. Apropos this issue, the type of glomerular-binding antibodies present in the patients who were analyzed in the current study recapitulated their underlying antichromatin response. Our observations that lupus nephritis patients can be divided into two groups, depending both on their GBM-binding IgG and their overall antichromatin response, parallel other recent studies on the underlying autoimmune response in lupus (15). In both studies, SLE patients could be segregated into two groups: one with predominant reactivity with chromatin, subnucleosomes, and dsDNA, and one with predominant reactivity with chromatin and subnucleosomes. The former group serologically resembles MRL lpr mice with established autoimmunity, whereas the latter group resembles young MRL lpr mice at the inception of autoimmunity, suggesting that these groups of patients may represent different stages in the evolution of the breakdown of tolerance $(14,16)$.

In addition to supporting the concept that multiple autoantibodies contribute to lupus nephritis, the current data are most consonant with either the planted antigen or immune complex hypotheses for lupus nephritis (1). Most of IgG binding to GBM in lupus nephritis patients was abolished by DNase pretreatment of GBM extracts, reconstituted with nuclear antigens after DNase, and inhibited by exogenous nuclear antigens similar to what we have observed in murine lupus $(24,25)$. These data demonstrate that the autoantibodies detected by the GBM ELISA can form immune complexes with nuclear antigens trapped by the GBM. It is nonetheless possible that GBM-binding IgG may complex with nucleosomes in the circulation and then deposit in the GBM. Nucleosome/antinucleosome complexes have been shown to bind avidly to the glomerulus in vivo and to the $\operatorname{GBM}$ in vitro $(18,20$, $25)$. Although our data suggest that most GBM-binding IgG is not comprised by such complexes (GBM binding was stable to DNase/high ionic strength), we cannot exclude a contribution of these complexes to GBM binding. In fact, our murine data suggest the presence of small amounts of immune complexes with glomerular-binding activity (22). The relatively low level of such complexes, however, may be a function of rapid clearance.

The current data do not support a major contribution of antibodies that bind directly to glomeruli (e.g., cross-reactive anti-DNA antibodies, autoantibodies to GBM proteins [3841]), although the GBM ELISA may not detect autoantibodies to intrinsic glomerular cells (42). Of relevance to this issue are recent studies that have cast doubt on the existence of such autoantibodies. The binding of cross-reactive anti-DNA mAbs to nonnuclear antigens or cells appears to be indirect in many circumstances and mediated by immune complexes that are formed in vitro with nucleosomes $(20,43)$. Nuclear antigens also bind avidly to basement membrane components isolated from biological sources (as we have noted with our GBM preparations [24]), and cells appear to release nucleosomes during in vitro culture that may adhere to cell surfaces and the pericellular matrix $(44,45)$. These adherent nuclear antigens may be responsible for the binding interactions observed with autoantibodies and various basement membrane proteins or cells. 
It is of note that lupus sera bound differently to GBM compared with placental ECM (without or with nucleosomes), despite the overall similarity in protein composition. This divergence may be a function of finer differences in the composition of the GBM relative to other ECMs (e.g., in the $\alpha$ chain composition of type IV collagen), as well as the nuclear antigens that adhere to GBM relative to other ECMs. Further investigation of how nuclear antigens interact with the ECM thus would appear relevant to the pathogenesis of lupus nephritis.

Germane to this issue, the present study specifically suggests that GBM collagen (the major GBM protein [46]) is important for autoantibody binding to the glomerulus in lupus nephritis, as we have noted with murine lupus sera and glomerulotropic mAbs $(24,25)$. Unlike murine lupus, however, we noted substantial variability between patients, suggesting that GBM proteins other than collagen (e.g., heparan sulfate proteoglycan [20]) probably play an important role in autoantibody binding to the glomerulus. The collagen dependence of GBM binding is likely to be a function of the avidity of histones for type IV collagen (35) and does not appear to be caused by the binding of sera to collagen per se. We also observed that the degree of collagen dependence of binding relates to the level, and consequently, type of GBM binding IgG (i.e., DNA/nucleosomal vs. nucleosomal) that also correlates with the patient's clinical status. Thus, nuclear antigen specificity, GBM binding properties, and pathogenicity of autoantibodies appear to be interrelated variables.

The current work further supports the conclusion that GBM-binding IgG in lupus is pathogenic and necessary for the development of nephritis. Elevated levels were noted in virtually all lupus patients with nephritis, adequate responses to therapy in these patients were accompanied by significant reductions in antibody levels, and particularly high levels of GBM-binding IgG were associated with more severe clinical disease. The presence of GBM-binding IgG, however, is clearly not sufficient for the development of nephritis. As noted, GBM-binding IgG was observed in patients with active nonrenal lupus, symptomatic drug-induced lupus, and occasionally in inactive lupus. In addition, neither the level nor the overall characteristics of GBM-binding IgG in nephritis patients could be related to the renal histopathology or prognosis in a simple fashion. Similar disparities between the autoantibody response and both the presence and severity of nephritis, while not apparent within the inbred strains of murine lupus, are nonetheless apparent in interspecific back-crosses of MRL lpr mice and in the graft vs. host model of murine lupus when induced across varying MHC class II differences $(47,48)$.

Therefore, we would conclude that glomerular binding IgG is necessary but not sufficient for the development of nephritis. The development of nephritis and specific histopathologic presentations, as well as the propensity of glomerular inflammation to progress to glomerulosclerosis, must result from a more complex array of factors. Conjecturally, specific antibodies within the broad categories defined by our GBM assay may be more nephritogenic than others. This capacity may simply be a function of the IgG subclass (and the ability to fix complement) or may be a function of the epitopes of chromatin that are most often accessible when adherent to the GBM in vivo. Additionally, factors relating to the release and clearance of chromatin, as well as the formation of circulating immune complexes, may contribute to the development of nephritis and specific pathologic variants. Finally, genetic variables con- trolling the extent of the local inflammatory response may contribute to the severity and long-term outcome of nephritis. Resolution of these issues will be critical in refining current diagnostic tests, improving prognostication, and targeting therapy in a more specific fashion.

In conclusion, the current study demonstrates the heterogeneity inherent in glomerulotropic antibodies in patients with lupus nephritis and argues against an exclusive role for antiDNA antibodies in this disorder. Specifically, the data suggest that the pathogenesis of nephritis results from the binding of antibodies to various epitopes on chromatin that bind to GBM largely (though not exclusively) via collagen. Although GBMbinding IgG levels are correlated to the concurrent clinical status of the patient and vary with the therapeutic response, the genesis of nephritis and its various pathologic forms and the factors controlling the long-term outcome of nephritis remain to be clarified.

\section{Acknowledgments}

This work was funded by a grant from the Arthritis Foundation. Drs. Di Valerio and Bernstein are supported by postdoctoral fellowship grants from the Arthritis Foundation.

\section{References}

1. Lefkowith, J.B., and G.S. Gilkeson. 1996. Nephritogenic autoantibodies in lupus: current concepts and continuing controversies. Arth. Rheum. 39:894-903. 2. Andrews, B.S., R.A. Eisenberg, A.N. Theofilopoulos, S. Izui, C.B. Wilson, P.J. McConahey, E.D. Murphy, J.B. Roths, and F.J. Dixon. 1978. Spontaneous murine lupus-like syndromes. Clinical and immunopathological manifestations in several strains. J. Exp. Med. 148:1198-1215.

3. Hecht, B., N. Siegel, M. Adler, M. Kashgarian, and J.P. Hayslett. 1976 Prognostic indices in lupus nephritis. Medicine. 55:163-181.

4. Koffler, D., P.H. Schur, and H.G. Kunkel. 1967. Immunological studies concerning the nephritis of systemic lupus erythematosus. J. Exp. Med. 126: $607-624$.

5. Ebling, F., and B.H. Hahn. 1980. Restricted subpopulations of DNA antibodies in kidneys of mice with systemic lupus: comparison of antibodies in serum and renal eluates. Arth. Rheum. 23:392-403.

6. Lambert, P.H., and F.J. Dixon. 1968. Pathogenesis of the glomerulonephritis of NZB/W mice. J. Exp. Med. 127:507-521.

7. Vlahakos, D.V., M.H. Foster, S. Adams, M. Katz, A.A. Ucci, K.J. Barrett, S.K. Datta, and M.P. Madaio. 1992. Anti-DNA antibodies form immune deposits at distinct glomerular and vascular sites. Kidney Int. 41:1690-1700.

8. Ohnishi, K., F.M. Ebling, B. Mitchell, R.R. Singh, B.H. Hahn, and B.P. Tsao. 1994. Comparison of pathogenic and non-pathogenic murine antibodies to DNA-antigen binding and structural characteristics. Int. Immunol. 6:817830.

9. Gilkeson, G.S., K.A. Bernstein, A.M.M. Pippin, S.H. Clark, T. Marion, D.S. Pisetsky, P. Ruiz, and J.B. Lefkowith. 1995. The influence of variable region somatic mutations on the specificity and pathogenicity of murine monoclonal anti-DNA antibodies. Clin. Immunol. Immunopathol. 76:59-67.

10. Yoshida, H., A. Kohno, K. Ohta, S. Hirose, N. Maruyama, and T. Shirai. 1981. Genetic studies of autoimmunity in New Zealand mice. III. Associations among anti-DNA antibodies, NTA, and renal disease in $(\mathrm{NZB} \times \mathrm{NZW}) \mathrm{F}_{1} \times$ NZW backcross mice. J. Immunol. 127:433-437.

11. Izui, S., V.E. Kelley, K. Masuda, H. Yoshida, J.B. Roths, and E.D. Murphy. 1984. Induction of various autoantibodies by mutant gene lpr in several strains of mice. J. Immunol. 133:227-233.

12. Lloyd, W., and P.H. Schur. 1981. Immune complexes, complement, and anti-DNA in exacerbations of systemic lupus erythematosus (SLE). Medicine. 60:208-217.

13. Appel, A.E., L.B. Sablay, R.A. Golden, P. Barland, A.I. Grayzel, and N Bank. 1978. The effect of normalization of serum complement and anti-DNA antibody on the course of lupus nephritis. Am. J. Med. 64:274-283.

14. Burlingame, R.W., R.L. Rubin, R.S. Balderas, and A.N. Theofilopoulos 1993. Genesis and evolution of antichromatin autoantibodies in murine lupus implicates T-dependent immunization with self antigen. J. Clin. Invest. 91:16871696.

15. Burlingame, R.W., M.L. Boey, G. Starkebaum, and R.L. Rubin. 1994. The central role of chromatin in autoimmune responses to histones and DNA in systemic lupus erythematosus. J. Clin. Invest. 94:184-192.

16. Amoura, Z., H. Chabre, S. Koutouzov, C. Lotton, A. Cabrespines, J.F. 
Bach, and L. Jacob. 1994. Nucleosome-restricted antibodies are detected before anti-dsDNA and/or antihistone antibodies in serum of MRL-Mp lpr/lpr and +/+ mice, and are present in kidney eluates of lupus mice with proteinuria. Arth. Rheum. 37:1684-1688.

17. Mohan, C., S. Adams, V. Stanik, and S.K. Datta. 1993. Nucleosome: a major immunogen for pathogenic autoantibody-inducing $\mathrm{T}$ cells of lupus. $J$. Exp. Med. 177:1367-1381.

18. Tax, W.J.M., C. Kramers, M.C.J. Vanbruggen, and J.H.M. Berden. 1995. Apoptosis, nucleosomes, and nephritis in systemic lupus erythematosus. Kidney Int. 48:666-673.

19. Chabre, H., Z. Amoura, J.-C. Piette, P. Godeau, J.-F. Bach, and S. Koutouzov. 1995. Presence of nucleosome-restricted antibodies in patients with systemic lupus erythematosus. Arth. Rheum. 38:1485-1491.

20. Kramers, C., M.N. Hylkema, M.C.J. Vanbruggen, R. Vandelagemaat, H.B.P.M. Dijkman, K.J.M. Assmann, R.J.T. Smeenk, and J.H.M. Berden. 1994. Antinucleosome antibodies complexed to nucleosomal antigens show antiDNA reactivity and bind to rat glomerular basement membrane in vivo. J. Clin. Invest. 94:568-577.

21. Monestier, M., K.E. Novick, E.T. Karam, L. Chabanne, J.C. Monier, and D. Rigal. 1995. Autoantibodies to histone, DNA and nucleosome antigens in canine systemic lupus erythematosus. Clin. Exp. Immunol. 99:37-41.

22. Bernstein, K.A., D. Bolshoun, G. Gilkeson, T. Munns, and J.B. Lefkowith. 1993. Detection of glomerular-binding immune elements in murine lupus using a tissue-based ELISA. Clin. Exp. Immunol. 91:449-455.

23. Bernstein, K.A., D. Bolshoun, and J.B. Lefkowith. 1993. Serum glomerular binding activity is highly correlated with renal disease in MRL lpr mice. Clin. Exp. Immunol. 93:418-423.

24. Bernstein, K.A., R. Di Valerio, and J.B. Lefkowith. 1995. Glomerular binding activity in MRL lpr serum consists of antibodies that bind to a DNA/histone/type IV collagen complex. J. Immunol. 154:2424-2433.

25. Di Valerio, R., K.A. Bernstein, E. Varghese, and J.B. Lefkowith. 1995. Murine lupus glomerulotropic monoclonal antibodies exhibit differing specificities but bind via a common mechanism. J. Immunol. 155:2258-2268.

26. Bernstein, K.A., L.E. Kahl, J.E. Balow, and J.B. Lefkowith. 1994. Serologic markers of lupus nephritis in patients: use of a tissue-based ELISA and evidence for immunopathogenic heterogeneity. Clin. Exp. Immunol. 98:60-65.

27. Tan, E.M., A.S. Cohen, J.F. Fries, A.T. Masi, D.J. McShane, N.F. Rothfield, J.G. Schaller, N. Talal, and R.J. Winchester. 1982. The 1982 revised criteria for the classification of systemic lupus erythematosus (SLE). Arthritis Rheum. 25:1271-1277.

28. Rubin, R.L., R.W. Burlingame, J.E. Arnott, M.C. Totoritis, E.M. McNally, and A.D. Johnson. 1995. IgG but not other classes of anti-[(H2A-H2B)DNA] is an early sign of procainamide-induced lupus. J. Immunol. 154:2483-2493.

29. Rubin, R.L., S.A. Bell, and R.W. Burlingame. 1992. Autoantibodies associated with lupus induced by diverse drugs target a similar epitope in the (H2A-H2B)-DNA complex. J. Clin. Invest. 90:165-173.

30. Gourley, M., H. Austin, C. Yarboro, E. Vaughan, J. Muir, M. Lindahl, D. Boumpas, D. Scott, J. Klippel, J. Balow, and A. Steinberg. 1995. Randomized trial of multiple boluses of methylprednisolone or cycloophosphamide or the combination in patients with lupus nephritis. Arth. Rheum. 38:392. (Abstr.)

31. Malide, D., I. Londono, P. Russo, and M. Bendayan. 1993. Ultrastructural localization of DNA in immune deposits of human lupus nephritis. Am. J. Pathol. 143:304-311.

32. Schmiedeke, T., F. Stoeckl, S. Muller, Y. Sugisaki, S. Batsford, R. Woitas, and A. Vogt. 1992. Glomerular immune deposits in murine lupus models may contain histones. Clin. Exp. Immunol. 90:453-458.
33. Stockl, F., S. Muller, S. Batsford, T. Schmiedeke, R. Waldherr, K. Andrassy, Y. Sugisaki, K. Nakabayashi, T. Nagasawa, B. Rodrigueziturbe, et al 1994. A role for histones and ubiquitin in lupus nephritis? Clin. Nephrol. 41:1017.

34. Schmiedeke, T.M.J., F.W. Stockl, R. Weber, Y. Sugisaki, S.R. Batsford and A. Vogt. 1989. Histones have high affinity for the glomerular basemen membrane: relevance for immune complex formation in lupus nephritis. J. Exp. Med. 169:1879-1894.

35. Bernstein, K.A., and J.B. Lefkowith. 1996. Histones bind to type IV collagen and, with DNA, form a unique ternary complex recognized by lupus autoantibodies. J. Invest. Med. 44:221A.

36. Burlingame, R.W., and R.L. Rubin. 1990. Subnucleosome structures as substrates in enzyme-linked immunosorbent assays. J. Immunol. Methods. 134: 187-199.

37. Brinkman, K., R.M. Termaat, J. de Jong, H.G. van den Brink, J.H.M Berden, and R.J.T. Smeenk. 1989. Cross-reactive binding patterns of monoclonal antibodies to DNA are often caused by DNA/anti-DNA immune complexes. Res. Immunol. 140:595-612.

38. Faaber, P., T.P.M. Rijke, L.B.A. van de Putte, P.J.A. Capel, and J.H.M. Berden. 1986. Cross-reactivity of human and murine anti-DNA antibodies with heparan sulfate. J. Clin. Invest. 77:1824-1830.

39. Raz, E., M. Brezis, E. Rosenmann, and D. Eilat. 1989. Anti-DNA antibodies bind directly to renal antigens and induce kidney dysfunction in the isolated perfused rat kidney. J. Immunol. 142:3076-3082.

40. Madaio, M.P., J. Carlson, J. Cataldo, A. Ucci, P. Migliorini, and O. Pankewycz. 1987. Murine monoclonal anti-DNA antibodies bind directly to glomerular antigens and form immune deposits. J. Immunol. 138:2883-2889.

41. Foster, M.H., J. Sabbaga, S.R.P. Line, K.S. Thompson, K.J. Barrett, and M.P. Madaio. 1993. Molecular analysis of spontaneous nephrotropic anti-laminin antibodies in an autoimmune MRL-lpr/lpr mouse. J. Immunol. 151:814-824.

42. Jacob, L., M.A. Lety, D. Louvard, and J.F. Bach. 1985. Binding of a monoclonal anti-DNA autoantibody to identical protein(s) present at the surface of several human cell types involved in lupus pathogenesis. J. Clin. Invest. 75:315-317.

43. Jacob, L., J.P. Viard, B. Allenet, M.F. Anin, F.B.H. Slama, J. Vandekerckhove, J. Primo, J. Markovits, F. Jacob, J.F. Bach, et al. 1989. A monoclonal anti-double stranded DNA autoantibody binds to a 94-kDa cell-surface protein on various cell types via nucleosomes or a DNA-histone complex. Proc. Natl. Acad. Sci. USA. 86:4669-4673.

44. Emlen, W., J. Niebur, and R. Kadera. 1994. Accelerated in vitro apoptosis of lymphocytes from patients with systemic lupus erythematosus. J. Immunol. 152:3685-3692.

45. Bell, D.A., B. Morrison, and P. VandenBygaart. 1990. Immunogenic DNA-related factors. Nucleosomes spontaneously released from normal murine lymphoid cells stimulate proliferation and immunoglobulin synthesis of normal mouse lymphocytes. J. Clin. Invest. 85:1487-1496.

46. Paulsson, M. 1992. Basement membrane proteins—structure, assembly, and cellular interactions. Crit. Rev. Biochem. Mol. Biol. 27:93-127.

47. Watson, ML., JK Rao, G.S Gilkeson, P Ruiz, E.M. Eicher, D.S. Pisetsky, A. Matsuzawa, J.M. Rochelle, and M.F. Seldin. 1992. Genetic analysis of MRL-lpr mice: relationship of the Fas apoptosis gene to disease manifestations and renal disease-modifying loci. J. Exp. Med. 176:1645-1656.

48. Bradley, D.S., J.C. Jennette, P.L. Cohen, and R.A. Eisenberg. 1994. Chronic graft versus host disease-associated autoimmune manifestations are independently regulated by different MHC class II loci. J. Immunol. 152:19601969. 